\title{
Understanding past changes in sea ice in the Southern Ocean
}

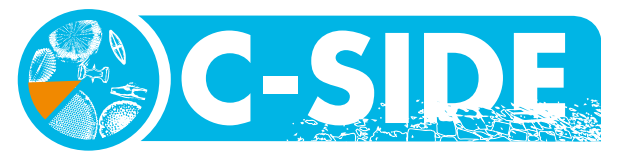

\author{
Rachael Rhodes ${ }^{1}$, K. Kohfeld ${ }^{2}$, H. Bostock ${ }^{3}$, X. Crosta ${ }^{4}$, A. Leventer ${ }^{5}$, \\ K. Meissner ${ }^{6}$ and O. Esper \\ 1st C-SIDE workshop, Vancouver, Canada, 24-26 October 2018
}

C-SIDE (pastglobalchanges.org/ini/wg/cside/intro) aims to reconstruct changes in Antarctic sea-ice extent in the Southern Ocean over the past 130,000 years and to understand the impact of sea ice on processes such as productivity, nutrient cycling, ocean circulation, carbon cycle, and air-sea gas exchange.

At this inaugural workshop, 32 participants (including three who joined remotely) from 11 countries brought together a broad spectrum of complementary expertise from proxy-based sea-ice reconstructions to Earth system modeling. The two-day program (pastglobalchanges.org/ini/wg/cside/meetings/127-pages/1812-c-side-wshopoct-18) featured presentations, speed talks, break-out groups and plenary discussions. A particular highlight was a well-attended evening public presentation at the SFU Harbour Centre followed by a public Q\&A session.

Workshop participants presented the current understanding of paleo proxies used to reconstruct Antarctic sea-ice conditions, including fossil diatom assemblages, highly-branched isoprenoid biomarkers, and ice-core chemical constituents. All these proxies have great potential as indicators of past sea-ice changes but each has its limitations. Participants considered how various proxy information could be combined to provide complementary evidence of sea-ice changes and described ongoing needs for ground-truthing, culture experiments and process-based modeling to improve our understanding of what each proxy represents.

\section{Presentations on past Antarctic sea-ice} changes demonstrated a need for improved temporal resolution and spatial coverage of sea-ice records. However, participants also identified a substantial amount of exciting, ongoing work that will partly help fill these data gaps. One critical target is the ability to reconstruct summer sea-ice extent and to constrain the seasonal cycle of sea ice because it directly impacts deep-water formation, primary production, and air-sea gas exchange. Workshop participants suggested that ongoing and published work be integrated to develop a series of Southern Ocean transects that cross oceanographic fronts and include both ice and sediment cores.

Complementary presentations described improvements in model representation of present-day sea ice and the difficulty in capturing spatial heterogeneity of sea-ice extent around Antarctica. At the same time, Earth system model transient simulations can provide a dynamic framework for identifying how physical circulation (e.g. Southern Hemisphere westerly winds and deepwater formation) as well as biogeochemical processes (e.g. nutrient and carbon cycling) both in and beyond the Southern Ocean are linked to Antarctic sea-ice changes.

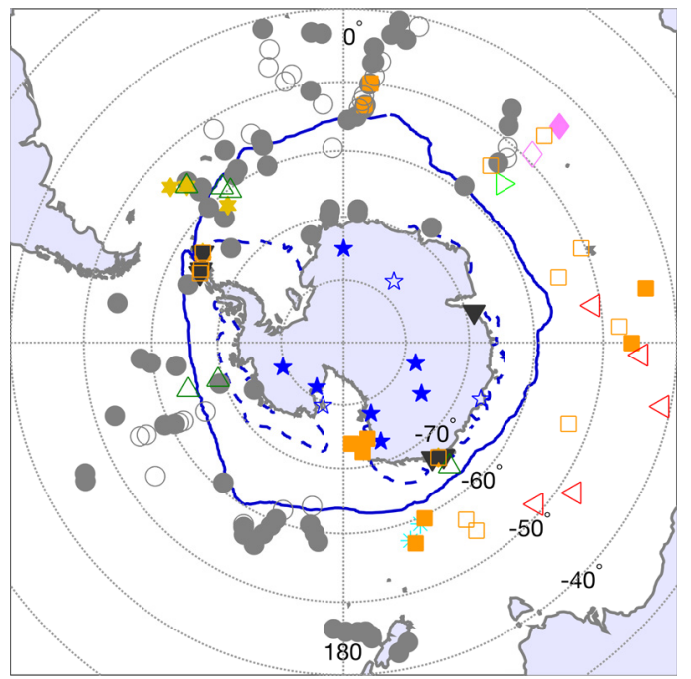

Figure 1: Locations of 160 sites identified by C-SIDE workshop participants with published (closed) and unpublished (open) proxy data that can be used for reconstructing sea-ice characteristics over the past 130,000 years. Sites are identified by H. Bostock \& K. Kohfeld (cyan asterisks), M. Chadwick (dark green triangles), O. Esper (grey circles), P. Ghadi (green sideways triangle); J. Mueller (gold stars), A. Nair \& X. Crosta (pink diamonds), M. Patterson \& A. Leventer (red sideways triangles), X. Crosta (orange squares), X. Crosta \& J. Mueller (black inverted triangles), and R. Rhodes (blue stars). Dark blue solid and dashed lines represent the 1981-2010 median sea-ice extent in September and February, respectively (taken from National Snow and Ice Data Center, nsidc.org/data/seaice_index/archives/)

The last full glacial-interglacial cycle is a key interval of focus because: (a) it offers the chance to explore how Antarctic sea ice responded during periods of warmer temperatures than today; and (b) it includes major climate transitions from both warm-tocold and cold-to-warm climate states, which may shed light on the pace of sea-ice/climate responses, as well as on the potential reversibility of these responses.

Researchers involved in developing proxies agreed upon a framework with which to share and consolidate available datasets that will ultimately be used to produce consistent reconstructions. Likewise, modelers decided how to synthesize sea-ice and sea-surface temperature simulations from the paleoclimate modeling projects PMIP3 and PMIP4 (when these become available in 2019). Collating complementary datasets that can be used to quantify the interaction of sea ice with the wider climate system was recognized as an important objective. A presentation and Q\&A session with developers of the Linked Paleo Data (lipd.net) facility sparked interest and discussion around issues of data sharing.

A primary objective of the workshop is to produce a review paper that synthesizes the current knowledge of past Antarctic sea-ice changes and their impact on the climate system. The next C-SIDE workshop is planned for 29-31 August 2019, just prior to the International Conference on Paleoceanography in Sydney, Australia. It will focus on synthesizing and reconciling the various sea-ice proxies and model simulations across the entire glacial cycle. We thank PAGES and Simon Fraser University, Canada, for financial support.

\section{AFFILIATIONS}

'Department of Earth Sciences, University of Cambridge, UK, now at: Department of Geography \& Environmental Sciences, Northumbria University, Newcastle upon Tyne, UK

${ }^{2}$ School of Resource and Environmental Management, Simon Fraser University, Burnaby, Canada ${ }^{3}$ National Institute of Water and Atmospheric Research, Wellington, New Zealand

${ }^{4}$ Oceanic and Continental Environments and Paleoenvironments, University of Bordeaux, France ${ }^{5}$ Department of Geology, Colgate University, Hamilton, NY, USA

${ }^{6}$ Climate Change Research Centre, University of New South Wales, Sydney, Australia

${ }^{7}$ Alfred-Wegener Institute for Polar and Marine Research, Bremerhaven, Germany

CONTACT

Karen Kohfeld: kohfeld@sfu.ca 